\title{
Ultimate braid-group generators for coordinate exchanges of Ising anyons from the multi-anyon Pfaffian wave functions
}

\author{
Lachezar S. Georgiev \\ Institut für Mathematische Physik, Technische Universität Braunschweig, Mendelssohnstr. 3, \\ 38106 Braunschweig, Germany, \\ Institute for Nuclear Research and Nuclear Energy, Bulgarian Academy of Sciences, \\ Tsarigradsko Chaussee 72, 1784 Sofia, Bulgaria
}

\begin{abstract}
We give a rigorous and self-consistent derivation of the elementary braid matrices representing the exchanges of adjacent Ising anyons in the two inequivalent representations of the Pfaffian quantum Hall states with even and odd number of Majorana fermions. To this end we use the distinct operator product expansions of the chiral spin fields in the Neveu-Schwarz and Ramond sectors of the two-dimensional Ising conformal field theory. We find recursive relations for the generators of the irreducible representations of the braid group $\mathscr{B}_{2 n+2}$ in terms of those for $\mathscr{B}_{2 n}$, as well as explicit formulas for almost all braid matrices for exchanges of Ising anyons. Finally we prove that the braid-group representations obtained from the multi-anyon Pfaffian wave functions are completely equivalent to the spinor representations of $\mathrm{SO}(2 n+2)$ and give the equivalence matrices explicitly. This actually proves that the correlation functions of $2 n$ chiral Ising spin fields $\sigma$ do indeed realize one of the two inequivalent spinor representations of the rotation group $\mathrm{SO}(2 n)$ as conjectured by Nayak and Wilczek.
\end{abstract}

PACS numbers: 71.10.Pm, 73.43.-f, 03.67.Lx

Keywords: Topological quantum computation, Conformal field theory, Non-Abelian statistics

\section{Introduction}

One fascinating application [1, 2] of the anticipated non-Abelian statistics of the chiral spin fields in the critical two-dimensional Ising model has established a remarkable connection between the rich-of-exact-results area of the two-dimensional rational conformal field theories (CFT) and the new and promising field of topological quantum computation [3]. The localized non-Abelian Ising anyons, which are believed to be realized in the fractional quantum Hall state at filling factor $v=5 / 2$, that is most likely described by the Moore-Read (Pfaffian) CFT [4], turned out to be a useful tool for topologically protected quantum information processing [1, 2, 5, 3]. Protection against noise and decoherence is obtained by encoding quantum information in robust topological characteristics of the strongly correlated electron system, such as quasiparticle's fusion channels, while quantum gates are implemented by topologically non-trivial operations [1, 2, 5, 6, such as braidings of non-Abelian anyons.

Nayak and Wilczek argued in an insightful paper that the Pfaffian wave functions with $2 n$ Ising anyons at fixed positions belong to a $2^{n-1}$ dimensional spinor representation of the rotation group $\mathrm{SO}(2 n)$, see Sect. 9 in Ref. [7]. However, as explained in Sect. 1.3 below, the arguments they presented in support of this claim were incomplete and partly misleading. To our knowledge, this claim therefore has been, up to now, only a conjecture. In the present 
paper we provide a complete proof of this conjecture meeting the requirements of rigor of mathematics. This proof contains three steps:

(i) Derive the elementary generators, $B_{j}^{(4, \pm)}, j=1,2,3$, of the braid group $\mathscr{B}_{4}$, in the two inequivalent irreducible representations with positive ("+" in the superscript) and negative ("-" in the superscript) fermion parity, directly from the 4-quasihole Pfaffian wave functions.

(ii) Construct recursively the generators $B_{j}^{(2 n+2, \pm)}, 1 \leq j \leq 2 n+1$, of the irreducible representations of the braid group $\mathscr{B}_{2 n+2}$ in terms of the generators, $B_{j}^{(2 n, \pm)}, 1 \leq j \leq$ $2 n-1$, of $\mathscr{B}_{2 n}$.

(iii) Find explicitly the equivalence matrices mapping the obtained generators of the representations of the braid group $\mathscr{B}_{2 n+2}$ to those generating the representations of Nayak-Wilczek with the corresponding parity.

Contrary to the common believe, the first step has not been accomplished by NW in Ref. [7]they have only computed the first line of the $2 \times 2$ matrix representing the exchange of the anyons with coordinates $\eta_{1}$ and $\eta_{3}$, which at best could be used to determine the generators of the positive-parity representation of $\mathscr{B}_{4}$, though some ambiguities had to be resolved, see Sect. 1.3 below. Later the braid matrix $R_{23}$, as well as the other two generators $R_{12}$ and $R_{34}$, for the exchanges of 4 Ising anyons have been unambiguously derived in Ref. [5] by careful analytic continuation of the 4-quasiholes Pfaffian wave functions in the representation corresponding to even number of fermion fields in the CFT correlation function.

However, the generators of the negative-parity representation of $\mathscr{B}_{4}$ have never been derived before, in the wave-function approach, because the 4-anyon Pfaffian wave functions with odd number of Majorana fermions have been unknown. In this paper we give the first, to our knowledge, derivation of the generators of the negative-parity representation of $\mathscr{B}_{4}$ directly from the Pfaffian wave functions, without computing them explicitly, by using instead the short-distance operator product expansions.

Furthermore, the generators of the two inequivalent representations of the braid group $\mathscr{B}_{2 n+2}$, spanned by the Pfaffian wave functions realized as CFT correlation functions with $2 n+2$ Ising anyons at fixed positions and even/odd number of Majorana fermions, are not easy to obtain. They cannot be derived in analogy with the generators of $\mathscr{B}_{4}$ because the Pfaffian wave functions with $2 n+2$ anyons are not known explicitly. Fortunately, it is possible to find recursion relations between the generators of $\mathscr{B}_{2 n+2}$ and those of $\mathscr{B}_{2 n}$, by using the fusion rules of the Ising anyons. However, when we fuse two Ising anyons, the result could be either $\mathbb{I}$ or a Majorana fermion $\psi$ so that in the first case the fusion process maps a representation of $\mathscr{B}_{2 n+2}$ with given fermion parity into a representation of $\mathscr{B}_{2 n}$ with the same parity, while in the second case the fusion process switches to the opposite parity. This subtlety not only mixes the representations but also requires that we know both representations of $\mathscr{B}_{2 n}$ in order to construct inductively from them any of the representations of $\mathscr{B}_{2 n+2}$.

Meanwhile, the 4-quasihole results of NW have been reproduced in Ref. [8] using the universal $R$ matrix in the quantum-group approach for the Ising model. It is worth stressing that the arguments of [8] do not prove the NW conjecture because the representation of the braid group $\mathscr{B}_{4}$ in [8] is defined by the authors in such a way to reproduce the results of NW as can be seen from Sects. 5.2 and 5.3 in [8], however, there is no proof that it is the same as the braid-group representation obtained by analytic continuation of the multi-anyon Pfaffian wave functions as obtained e.g. in [5].

In addition, the 4-anyon braid matrices have been convincingly derived in Ref. [9] for the case of the $p$-wave superconductor, which is known to be related to the Pfaffian state [10, 11]. 
While the question of the basis orthonormality in the $p$-wave superconductor has not been addressed in [9], it has been answered in [11]. The analysis of the $p$-wave superconductor is more conclusive about the braid generators, however, the connection to the Pfaffian FQH state is more elaborate, because only the large-distance behavior of the weak-pairing phase is related to the MR state [10].

The above mentioned confirmations would have been very nice if we had an independent proof of the NW conjecture, however, they are still not sufficient to prove this conjecture, because they are either defined on purpose to reproduce the NW results or are indirectly related to the many-body states of the electron system and the correspondence depends on many assumptions. Therefore, it would be useful to have an independent, self-consistent and rigorous derivation of the braid matrices directly from the Pfaffian wave functions representing the states containing multiple Ising anyons, which are actually used to define the qubits in TQC [1].

One more reason for this necessity is that the eventual experiments with the real quantum Hall systems would test the properties of the strongly correlated electron state that are encoded into the corresponding many-body wave function. Recall that the gauge-invariant quantity in the adiabatic transport exchanging Ising quasiholes is the product of the explicit monodromy (which can be computed in the CFT or quantum group approach) and the geometrical Berry phase [12, 13, 14] which is present only in the wave-function approach. This is a subtle point because whether the adiabatic transport of Ising anyons, along complete loops around each other, is indeed realized by spinor representations of the rotation group depends on the Berry connection of the trial wave functions. It has been argued that the actual holonomy, which is the physically observable quantity that we intend to use for topological quantum computation, is indeed equal to the monodromy of the Pfaffian wave functions because the Berry connection is trivial [12, 11, 13, 14], i.e., the only contribution comes from the ubiquitous Gaussian factor that is typical for charged particles in magnetic field and this geometrical contribution is simply the Aharonov-Bohm phase. Given that the multi-anyon trial wave functions are holomorphic, that would certainly be true if they could be proven to be orthonormal [13, 14]. While the first attempts [12] succeed in generalizing the analogy of (the overlap screening of) the Coulomb plasma, at least to the Pfaffian state with two anyons, a recent argument about the four-anyon case [14], which could be generalized to more anyons, seems to provide convincing evidence that the multi-anyon wave functions obtained in an appropriate CFT basis are indeed orthonormal. Therefore, it is now rather plausible that the holonomy of the multianyon Pfaffian wave functions is precisely given by the monodromy which could be obtained by analytic continuation, and this is what we shall use in this paper. Notice also that the Landau level mixing, which has important consequences for the physics of the quantum Hall state at filling factor $v=5 / 2$, would certainly modify [13] the exchange properties of the Ising anyons derived by the monodromies and this effect can only be analyzed in the wave-function approach.

The details in the explicit representation of the braid generators and the differences between their distinct realizations become more important when we try to implement various quantum gates and to estimate the computational power of the Ising-anyon TQC [5]. For example, it was possible to construct the CNOT gate [5] in terms of 7 elementary braidings, however this construction could not be generalized for systems with more anyons, i.e., the CNOT could not be embedded into systems with more qubits. The precise braid-generators analysis is crucial for answering such questions as whether it is possible to implement the entire Clifford-gate group purely by braiding or not, see Ref. [6] for the answer.

Outline of the paper: In this paper we give a rigorous and unified derivation of the braid matrices, representing the exchanges of Ising anyons, and a proof of the NW conjecture based 
on the wave-function approach, and this result is independent of the orthonormality of the CFT blocks used as a basis. Combined with the orthonormality results obtained in Ref. [14] this implies that the adiabatic transport of Ising anyons could indeed be used for topological quantum computation as proposed in [7, 1, 11, 14]. In Sect. 1.1] we review the subtle issue of the chiral fermion parity and its spontaneous breaking in the doubly degenerate Ramond sector of the Ising model due to the presence of the Majorana fermion zero mode, following Refs. [15, 16] and introducing their notation. This is necessary for the formulation of the short-distance operator product expansions of the spin fields $\sigma_{ \pm}$in the Ramond sector, derived in [15], which is the main tool for the computation of the non-diagonal braid matrices $B_{2}^{(4, \pm)}$ in Sects. 3.2 and 3.3 In Sect. 1.2 we recall the definitions of the 4-quasihole Pfaffian wave functions as correlation functions [4, 7] in the $\widehat{u(1)} \times$ Ising CFT containing four $\sigma$ fields and even number of Majorana fermions and introduce the notation for the computational states, as well as the encoding of quantum information, following Refs. [5, 6]. In Sect.1.3 we formulate the NW conjecture, which we intend to prove in this paper, and in Sect. 1.4 we announce the precise statement about the braid generators that we obtain in the Pfaffian wave-function representations of the braid group $\mathscr{B}_{4}$ with positive and negative parity, which is the first step towards the proof. Then in Sect. 2 we derive the diagonal braid matrices $B_{1}^{(4, \pm)}$ and $B_{3}^{(4, \pm)}$ by using the short-distance operator product expansion (OPE) in the Neveu-Schwarz (NS) sector of the Ising model, representing the elementary 4-anyon exchanges in the positiveparity (Sect. 2.11) and negative-parity (Sect. 2.2) wave-function representations. Next, in Sect. 3 we derive the non-diagonal braid matrices $B_{2}^{(4, \pm)}$, in the wave-function representations with positive parity (Sect. 3.2) and negative parity (Sect. 3.3), by using the short-distance OPE, this time in the Ramond (R) sector of the Ising model, which is reviewed in Sect. 3.1 following Ref. [15]. The braid generators $B_{j}^{(4,+)}, j=1,2,3$, obtained in the representation of $\mathscr{B}_{4}$ with positive fermion parity, coincide completely with those derived in Ref. [5] for the case when the homotopic condition $\left|\eta_{12} \eta_{34} / \eta_{13} \eta_{24}\right|<1$ of Ref. [5] is fulfilled. Notice that $B_{j}^{(4,+)}$ obtained here are explicitly different from those of NW. Furthermore, the braid generators $B_{j}^{(4,-)}, j=1,2,3$, for the representation of $\mathscr{B}_{4}$ with negative fermion parity, are important new results which have not been obtained before from the 4-quasihole Pfaffian wave functions because these wave functions were unknown in the negative-parity representations. In the Proposition 1 in Sect. 4 we derive new recursive relations for the projected $(2 n+2)$ anyon exchange generators $B_{j}^{(2 n+2, \pm)}, j=1, \ldots, 2 n+1$, in terms of those for $2 n$ anyons, $B_{j}^{(2 n, \pm)}, j=1, \ldots, 2 n-1$, and give maximally explicit new formulas for the (projected) braid matrices in the Corollary. Finally we prove in the Proposition 2 in Sect. 5 that the braid-group representations derived from the multi-anyon Pfaffian wave functions are equivalent to those derived in the $\mathrm{SO}(2 n+2)$ spinor approach and give the explicit equivalence matrices which are also new results. This equivalence makes it completely legitimate to interpret the abstract parity in the spinor representations of $\mathrm{SO}(2 n+2)$ as the physical fermion parity in the Ising model.

\subsection{Double degeneracy of the R-sector and spontaneous breaking of fermion parity}

The Ramond sector (or twisted sector) of the Ising model is defined as the superselection sector in which the Majorana fermion field has periodic boundary conditions on the cylinder and antiperiodic on the conformal plane [15, 17]. Because of the periodic boundary conditions 
of the Majorana fermion in the R-sector, whose Laurent mode expansion in the complex plane

$$
\psi(z)=\sum_{n \in \mathbb{Z}} \psi_{n} z^{n-1 / 2}, \quad\left\{\psi_{n}, \psi_{m}\right\}=\delta_{n+m, 0}
$$

implies the presence of a fermionic zero mode $\psi_{0}$, the ground state in this sector must necessarily be doubly degenerate if the chiral fermion parity is conserved. Indeed, the Majorana zero mode $\psi_{0}=\psi_{0}^{\dagger},\left(\psi_{0}\right)^{2}=(1 / 2) \mathbb{I}$ and the chiral fermion parity operator $\gamma$, satisfying $\gamma \psi_{0}+\psi_{0} \gamma=0$ and $\gamma^{2}=\mathbb{I}$, form a two-dimensional Clifford algebra whose lowest dimensional representation is two-dimensional and can be expressed in terms of the Pauli matrices [16]. Choosing a $\gamma$-diagonal basis, the two chiral spin fields of CFT dimension $1 / 16$ (with positive and negative fermionic parity) intertwining between the vacuum and the R-sector's lowest weight state can be written as [15]

$$
| \pm\rangle=\sigma_{ \pm}(0)|0\rangle, \quad \gamma \sigma_{ \pm} \gamma= \pm \sigma_{ \pm} .
$$

The conservation of the fermion parity implies that the two fields $\sigma_{ \pm}$in Eq. (1) must obey Abelian fusion rules

$$
\sigma_{+} \times \sigma_{+}=\mathbb{I}, \quad \sigma_{+} \times \sigma_{-}=\psi, \quad \sigma_{-} \times \sigma_{-}=\mathbb{I}, \quad \psi \times \sigma_{ \pm}=\sigma_{\mp} .
$$

On the other hand, modular invariance requires a single lowest-weight state [16], like in the case of the Gliozzi-Scherk-Olive projection in string theory, which is conventionally chosen as

$$
\sigma=\frac{\sigma_{+}+\sigma_{-}}{\sqrt{2}} \Longrightarrow \sigma \times \sigma=\mathbb{I}+\psi
$$

and consequently obeys non-Abelian statistics. This projection leads to spontaneous breaking of the chiral fermion parity, see Ref. [16] for a more detailed explanation. Despite the seemingly unphysical nature of the chiral spin fields $\sigma_{ \pm}$with definite fermion parity they appear to be very convenient for enumerating different computational states, for labeling the fusion paths in the corresponding Bratteli diagrams [3, 6], as well as for the identification of the spinor parity in the representations of $\mathrm{SO}(2 n+2)$ with the fermion parity in the Ising model.

\subsection{Wave functions for 4 Ising anyons in the positive-parity representation}

The wave function for the Pfaffian fractional quantum Hall state with even number $N$ of holes (or electrons) at positions $z_{1}, \ldots, z_{N}$ and four quasiholes at positions $\eta_{1}, \ldots, \eta_{4}$, can be realized as a correlation function, in the $\widehat{u(1)} \times$ Ising CFT [4, 7, 5],

$$
\Psi_{4 \mathrm{qh}}\left(\eta_{1}, \eta_{2}, \eta_{3}, \eta_{4} ;\left\{z_{i}\right\}\right)=\left\langle\psi_{\mathrm{qh}}\left(\eta_{1}\right) \psi_{\mathrm{qh}}\left(\eta_{2}\right) \psi_{\mathrm{qh}}\left(\eta_{3}\right) \psi_{\mathrm{qh}}\left(\eta_{4}\right) \prod_{i=1}^{N} \psi_{\text {hole }}\left(z_{i}\right)\right\rangle(3)
$$

of the field operators corresponding to creation of holes and quasiholes

$$
\psi_{\text {hole }}(z)=\psi(z): \mathrm{e}^{i \sqrt{2} \phi(z)}: \quad \text { and } \quad \psi_{\mathrm{qh}}(\eta)=\sigma(\eta): \mathrm{e}^{i \frac{1}{2 \sqrt{2}} \phi(\eta)}:,
$$

respectively, where $\sigma(\eta)$ is the chiral spin field in the Ising model of dimension $1 / 16$ and $\psi(z)$ is the right-moving Majorana fermion in the chiral Ising model. It can be expressed in more explicit form in terms of the Pfaffian wave functions [5], however, because they will not be needed in our fusion-rules approach we will skip them. We shall only use the notation of Ref. [7, 5] for the two linear independent 4-quasiholes states

$$
\Psi_{4 \mathrm{qh}}^{(0)} \equiv|0\rangle_{+} \sim\left\langle\sigma_{+} \sigma_{+} \sigma_{+} \sigma_{+}\right\rangle, \quad \Psi_{4 \mathrm{qh}}^{(1)} \equiv|1\rangle_{+} \sim\left\langle\sigma_{+} \sigma_{-} \sigma_{+} \sigma_{-}\right\rangle
$$


and will call them the computational basis in the positive-parity representation (representation parity is denoted by the subscript "+"). In the next section we will give more detailed expressions for the computational basis states (5) as well as an explanation of the sign " ". We can now argue why it is important to derive the braid generators directly from the wave functions representing FQH states with 4 Ising anyons. It might indeed be possible to derive the braid-group generators from the universal $R$-matrix for the quantum group corresponding to the Pfaffian FQH state [8]. However, it can be shown that the Pfaffian wave functions (5) with 4 Ising anyons, defined in Ref. [5], are different from the correlation functions of four $\sigma$ fields (which are zero unless $e_{1} e_{2}=e_{3} e_{4}=\kappa$ and then, see Eq. (6.43) in Ref. [15])

$$
\left\langle\sigma_{e_{1}}\left(\eta_{1}\right) \sigma_{e_{2}}\left(\eta_{2}\right) \sigma_{e_{3}}\left(\eta_{3}\right) \sigma_{e_{4}}\left(\eta_{4}\right)\right\rangle=\frac{1}{\sqrt{2}}\left(\frac{\eta_{13} \eta_{24}}{\eta_{12} \eta_{14} \eta_{23} \eta_{34}}\right)^{1 / 8} \sqrt{1+\kappa \sqrt{x}},(6)
$$

so that the former functions would have different analytic properties from the latter because of the presence of the Majorana fermions. Because the 4-point correlation functions (6) depend only on the product of the signs of the fields comprising the first and the second pair we can encode information in the topological charge $\kappa=e_{1} e_{2}$ of the first pair and then the topological charge of the second pair is fixed to be the same. It is also obvious from Eq. (6) that the order of signs in the two pairs is irrelevant, i.e., $\sigma_{+} \sigma_{-} \sim \sigma_{-} \sigma_{+}$because $e_{1} e_{2}=e_{2} e_{1}=\kappa$, so that we can always choose the sign of the first $\sigma$ field in each pair to be ' + '.

\subsection{The Nayak-Wilczek conjecture}

Nayak and Wilczek conjectured [7] that the elementary matrices representing the exchanges of $2 n$ Ising quasiparticles in the Pfaffian fractional quantum Hall state can be interpreted as $\pi / 2$ rotations from $\mathrm{SO}(2 n)$, i.e., they can be expressed in terms of the gamma matrices $\gamma_{i}^{(n)}$, $1 \leq i \leq 2 n$, which satisfy the anticommutation relations of the Clifford algebra

$$
\left\{\gamma_{i}^{(n)}, \gamma_{j}^{(n)}\right\}=2 \delta_{i j}, \quad 1 \leq i, j \leq 2 n
$$

In more detail, the elementary operations for the exchange of the $i$-th and $(i+1)$-th quasiparticles could be expressed, in an appropriate basis of $2 n$-quasiholes Pfaffian wave functions [7, 9], as

$$
R_{j}^{(n)}=\mathrm{e}^{i \frac{\pi}{4}} \exp \left(-\frac{\pi}{4} \gamma_{j}^{(n)} \gamma_{j+1}^{(n)}\right) \equiv \frac{\mathrm{e}^{i \frac{\pi}{4}}}{\sqrt{2}}\left(\mathbb{I}-\gamma_{j}^{(n)} \gamma_{j+1}^{(n)}\right)
$$

where $1 \leq j \leq 2 n-1$ and the second equality follows from the fact that $\left(\gamma_{j} \gamma_{j+1}\right)^{2}=-\mathbb{I}$ due to the anticommutation relations (7).

The $2 n$ matrices $\gamma_{i}^{(n)}$ have dimension $2^{n} \times 2^{n}$ and can be defined explicitly as follows $[18,7,8]$

$$
\begin{aligned}
\gamma_{1}^{(n)} & =\sigma_{1} \otimes \sigma_{3} \otimes \cdots \otimes \sigma_{3} \\
\gamma_{2}^{(n)} & =\sigma_{2} \otimes \sigma_{3} \otimes \cdots \otimes \sigma_{3} \\
& \vdots \\
\gamma_{2 j-1}^{(n)} & =\underbrace{\mathbb{I}_{2} \otimes \cdots \otimes \mathbb{I}_{2}}_{j-1} \otimes \sigma_{1} \otimes \underbrace{\sigma_{3} \otimes \cdots \otimes \sigma_{3}}_{n-j} \\
\gamma_{2 j}^{(n)} & =\underbrace{\mathbb{I}_{2} \otimes \cdots \otimes \mathbb{I}_{2}}_{j-1} \otimes \sigma_{2} \otimes \underbrace{\sigma_{3} \otimes \cdots \otimes \sigma_{3}}_{n-j}
\end{aligned}
$$




$$
\begin{aligned}
& \gamma_{2 n-1}^{(n)}=\mathbb{I}_{2^{n-1}} \otimes \sigma_{1} \\
& \gamma_{2 n}^{(n)}=\mathbb{I}_{2^{n-1}} \otimes \sigma_{2} .
\end{aligned}
$$

The "gamma-five" matrix $\gamma_{F}^{(n)}$, defined by

$$
\gamma_{F}^{(n)}=(-i)^{n} \gamma_{1}^{(n)} \cdots \gamma_{2 n}^{(n)}
$$

commutes with all matrices $\left[8\right.$ and therefore $R_{j}^{(n)}$ cannot change the $\gamma_{F}^{(n)}$ eigenvalues \pm 1 , which means that the representation (8) is reducible and the two irreducible components, corresponding to eigenvalues \pm 1 , can be obtained by projecting with the two projectors

$$
P_{ \pm}^{(n)}=\frac{\mathbb{I}_{2^{n}} \pm \gamma_{F}^{(n)}}{2} \text {, i.e., }\left(P_{ \pm}^{(n)}\right)^{2}=P_{ \pm}^{(n)}=\left(P_{ \pm}^{(n)}\right)^{\dagger} .
$$

In other words, the generators of the two irreducible spinor representations of the braid group $\mathscr{B}_{2 n}$ can be obtained by simply projecting $(1 \leq j \leq 2 n-1)$

$$
R_{j}^{(n, \pm)}=P_{ \pm}^{(n)} R_{j}^{(n)} P_{ \pm}^{(n)}=\frac{\mathrm{e}^{i \frac{\pi}{4}}}{\sqrt{2}}\left(\mathbb{I}-\gamma_{j}^{(n)} \gamma_{j+1}^{(n)}\right) P_{ \pm}^{(n)}
$$

Eq. (11) is what we call the NW conjecture in this paper because the braid generators (11) have not been derived in Ref. [7] from the multi-anyon Pfaffian wave functions. Instead, NW say on page 546 in [7]: "We will verify this assertion in the four-quasihole case with our explicit wave functions ... and give an argument in favor of its validity in the $2 n$-quasihole case". To this end they first verify the statement for 4 quasiholes by computing the first row of the $2 \times 2$ braid matrix representing the exchange $R_{13}$ of the anyons with coordinates $\eta_{1}$ and $\eta_{3}$ and then compute $R_{23}$ from that. However, this kind of derivation of the elementary braid matrix $R_{23}$ is ambiguous because it is based on the result for the exchange of anyons 1 and 3 and a braid relation, such as $R_{23}=R_{12} R_{13} R_{12}^{-1}$ or $R_{23}^{\prime}=R_{12}^{-1} R_{13} R_{12}$, and the two results are physically different. Formally, this ambiguity appears because the exchange $\eta_{1} \leftrightarrow \eta_{3}$ depends on the homotopy class of the exchange with respect to the second anyon (with coordinate $\eta_{2}$ ) and because of the emerging sign ambiguities which have not been fixed in a physical way. Next, the generalization argument they mention at the end of Sect. 9 in [7] is as follows: "... imagine bringing 4 quasiholes close together ...; the braiding is governed by the OPE and therefore is generated be the transformations we found above in the 4-quasihole case". This argument is misleading because NW found the 4-quasihole braid generators only for even fermion parity of "the rest", while their generalization argument assumes that they could use them also in the negative-parity case which is wrong. The point is that when we separate 4 quasiholes the rest $2 n-4$ quasiholes could have both positive and negative total fermion parity (with equal occurrence in the computational basis). For example, consider the 6-anyon computational states given in Eq. (38) in Ref. [5]: following the NW "generalization argument", let us concentrate on the first four quasiholes, corresponding to coordinates $\eta_{1}, \ldots, \eta_{4}$; the rest of the quasiholes, i.e., the two quasiholes with coordinates $\eta_{5}$ and $\eta_{6}$ in this case, have positive total fermion parity in the computational states denoted by $|00\rangle$ and $|10\rangle$ in Eq. (38) in [5], while in the states $|01\rangle$ and $|11\rangle$ it is negative. In the first case one could eventually use the four-quasihole results of Ref. [7], while for negative parity one needs an inequivalent set of generators which have been missing in [7]. Actually the braid generators in the negative-parity representations of the braid group $\mathscr{B}_{4}$ have never been known before, because the explicit four-anyon Pfaffian wave functions in the negative-parity representation have been unknown. Hence one cannot derive recursively the generators of 
$\mathscr{B}_{2 n}$ only from those of the positive-parity representation of $\mathscr{B}_{4}$, so that NW have drawn an inference from slight or insufficient evidence. In this paper we are filling this logical gap thus turning the insightful NW conjecture into a mathematical theorem.

\subsection{Braid matrices for exchanges of 4 Ising anyons: statement of the result}

One of the important results in this paper is the unified derivation of the braid matrices, $B_{1}^{(4, \pm)}$, $B_{2}^{(4, \pm)}$ and $B_{3}^{(4, \pm)}$, in the two inequivalent representations of the braid group $\mathscr{B}_{4}$ corresponding to positive and negative fermion parity, directly from the four-anyon Pfaffian wave functions. The superscript " 4 " in the notation for the braid generators expresses that these are generators of representations of $\mathscr{B}_{4}$, while the sign " \pm " denotes the fermion parity of the corresponding representation. In Sections 2 and 3 below we will show that the result for the generators of the positive-parity representation of $\mathscr{B}_{4}$

$$
B_{1}^{(4,+)}=\left[\begin{array}{ll}
1 & 0 \\
0 & i
\end{array}\right], \quad B_{2}^{(4,+)}=\frac{\mathrm{e}^{i \frac{\pi}{4}}}{\sqrt{2}}\left[\begin{array}{rr}
1 & -i \\
-i & 1
\end{array}\right], \quad B_{3}^{(4,+)}=\left[\begin{array}{ll}
1 & 0 \\
0 & i
\end{array}\right]
$$

obtained here by the fusion-rules approach completely coincides with the result of Ref. [19] obtained by the analytic continuation of the 4-quasiholes wave function when $\left|\eta_{12} \eta_{34} / \eta_{13} \eta_{24}\right|<1$.

The braid generators $B_{j}^{(4,+)}$ obtained here are explicitly different from those of NW, albeit the latter can be obtained by an equivalence transformation generated by $\left(B_{3}^{(4,+)}\right)^{2}$, see Sect. 4 below. However, this monodromy transformation makes an observable difference for the physical state the topological quantum computer, which has important physical consequences, e.g., it implements the Pauli $Z$ gate, and therefore has to be controlled experimentally.

In addition we shall explicitly derive the generators of the 4-quasihole Pfaffian representation of the braid group $\mathscr{B}_{4}$ with negative fermion parity

$$
B_{1}^{(4,-)}=\left[\begin{array}{ll}
1 & 0 \\
0 & i
\end{array}\right], \quad B_{2}^{(4,-)}=\frac{\mathrm{e}^{i \frac{\pi}{4}}}{\sqrt{2}}\left[\begin{array}{rr}
1 & -i \\
-i & 1
\end{array}\right], \quad B_{3}^{(4,-)}=\left[\begin{array}{ll}
i & 0 \\
0 & 1
\end{array}\right]
$$

which has not been obtained before in the wave-function approach.

The main idea is to employ the realization of the multi-anyon Pfaffian wave functions as CFT correlation functions without using their explicit form. The key point is that the precise braid matrices are independent of the distance between the particles being braided because they are topological objects. Therefore we could first fuse the particles, which we intend to exchange, and then execute braiding by analytic continuation of the relative coordinate. For example, the counterclockwise braiding of the quasiparticles with coordinates $\eta_{1}$ and $\eta_{2}$ could be executed by the analytic continuation along the circle defined by [5, 20]

$$
\eta_{1}^{\prime}=\frac{\eta_{1}+\eta_{2}}{2}+\mathrm{e}^{i \pi t} \frac{\eta_{12}}{2}, \quad \eta_{2}^{\prime}=\frac{\eta_{1}+\eta_{2}}{2}-\mathrm{e}^{i \pi t} \frac{\eta_{12}}{2}, \quad 0 \leq t \leq 1 .
$$

Thus, if we want to exchange the anyons with coordinates $\eta_{1}$ and $\eta_{2}$ we can first fuse $\eta_{1} \rightarrow \eta_{2}$ inside the CFT correlator, apply the OPE to extract the short-distance singular behavior in terms of the relative coordinate $\eta_{12}=\eta_{1}-\eta_{2}$, and then execute braiding by a simple permutation $\eta_{1} \leftrightarrow \eta_{2}$ plus analytic continuation in $\eta_{12}$, i.e.,

$$
\left.\eta_{1}^{\prime}=\eta_{2}, \quad \eta_{2}^{\prime}=\eta_{1}, \quad \text { (so that } \eta_{12}^{\prime}=\mathrm{e}^{i \pi} \eta_{12}\right), \quad \eta_{j}^{\prime}=\eta_{j}, \text { for } j>2 .
$$

This leads to crucial simplifications because the (potentially unknown) CFT correlators after fusion are independent of $\eta_{12}$ and their explicit form is not needed since the entire nonanalytic behavior comes from the short-distance prefactors containing $\eta_{12}$. 


\section{Diagonal exchange matrices $B_{1}^{(4, \pm)}$ and $B_{3}^{(4, \pm)}$ : fusion rules in the NS sector}

When we exchange the quasiholes with coordinates $\eta_{1}$ and $\eta_{2}$ or $\eta_{3}$ and $\eta_{4}$, corresponding to the braid generators $B_{1}^{(4, \pm)}$ and $B_{3}^{(4, \pm)}$ respectively, it is obvious from Eq. (3) that there are even or zero number of $\sigma$ fields to the right of the quasiholes being exchanged. Therefore, in order to fuse the quasiholes before braiding we can use the OPE of two $\sigma$ fields in the Neveu-Schwarz sector of the Ising model [15, 17].

The fusion rules for the $\sigma_{ \pm}$fields (2) lead to the following short-distance OPE in the NS sector [15, 17]

$$
\sigma_{ \pm}\left(z_{1}\right) \sigma_{ \pm}\left(z_{2}\right) \underset{z_{1} \rightarrow z_{2}}{\simeq} z_{12}^{-1 / 8} \mathbb{I}, \quad \sigma_{+}\left(z_{1}\right) \sigma_{-}\left(z_{2}\right) \underset{z_{1} \rightarrow z_{2}}{\simeq} z_{12}^{-1 / 8} \sqrt{\frac{z_{12}}{2}} \psi\left(z_{2}\right) .
$$

However there is another contribution to the OPE of the quasihole fields in (4) coming from the OPE of the Abelian parts of the quasiholes operators

$$
: \mathrm{e}^{i \frac{1}{2 \sqrt{2}} \phi\left(z_{1}\right)}:: \mathrm{e}^{i \frac{1}{2 \sqrt{2}} \phi\left(z_{2}\right)}: \underset{z_{1} \rightarrow z_{2}}{\simeq} z_{12}^{1 / 8}: \mathrm{e}^{i \frac{1}{\sqrt{2}} \phi\left(z_{2}\right)}:,
$$

which cancels the factors $z_{12}^{-1 / 8}$ and from now on we shall skip them (these factors have been explicitly shown as $\eta_{a b}^{1 / 8}$ in Eq. (9) in Ref. [5]).

It is worth-stressing that the braid matrices $B_{1}^{(4, \pm)}$ and $B_{3}^{(4, \pm)}$ must necessarily be diagonal [5] because the anyons being exchanged are in the NS sector where the chiral fermion parity is preserved [16] so no coherent superposition of states with different parity is possible. Indeed, if we want to exchange $\eta_{3}$ with $\eta_{4}$ we could first fuse them and then do the braiding. However, there are no other $\sigma$ fields to the right of the pair $\sigma\left(\eta_{3}\right) \sigma\left(\eta_{4}\right)$ so we have to use the OPE (14) in the NS sector and therefore, e.g., the matrix element ${ }_{+}\left\langle 0\left|B_{3}^{(4,+)}\right| 1\right\rangle_{+}$must be zero.

In the following subsections we will consider the two cases with positive and negative fermion parity separately.

\subsection{Positive-parity representation:}

Using the Abelian $\sigma_{ \pm}$fields with definite fermion parity and the fusion-path approach [3, 6] to label the anyonic states of matter we can write the computational basis (5) for 4 Ising anyons in the positive-parity representation as follows [19, 5, 21, 6]:

$$
\begin{aligned}
|0\rangle_{+} & \equiv\left\langle\sigma_{+}\left(\eta_{1}\right) \sigma_{+}\left(\eta_{2}\right) \sigma_{+}\left(\eta_{3}\right) \sigma_{+}\left(\eta_{4}\right) \prod_{j=1}^{2 N} \psi\left(z_{j}\right)\right\rangle \\
|1\rangle_{+} & \equiv\left\langle\sigma_{+}\left(\eta_{1}\right) \sigma_{-}\left(\eta_{2}\right) \sigma_{+}\left(\eta_{3}\right) \sigma_{-}\left(\eta_{4}\right) \prod_{j=1}^{2 N} \psi\left(z_{j}\right)\right\rangle .
\end{aligned}
$$

Recall that quantum information is encoded in the topological charge $\kappa$ of the first pair of $\sigma$ fields according to the rule $|0\rangle \leftrightarrow \sigma_{+} \sigma_{+},|1\rangle \leftrightarrow \sigma_{+} \sigma_{-}$, while the second pair of $\sigma$ fields carries no information - its purpose is to make the total fermion parity in (16) trivial, in order for the correlation functions to be nonzero, see Refs. [19, 5, 21, 6] for more detail.

To compute the braid matrix $B_{1}^{(4,+)}$, representing the exchange of the first two anyons, we can first fuse $\eta_{1} \rightarrow \eta_{2}$ and then implement braiding by $\eta_{12} \rightarrow \mathrm{e}^{i \pi} \eta_{12}$. The short-distance approximation of the two computational basis states are obtained by using the fusion rules (14) and (15)

$$
|0\rangle_{+} \underset{\eta_{1} \rightarrow \eta_{2}}{\stackrel{\sim}{\sim}}\left\langle\sigma_{+}\left(\eta_{3}\right) \sigma_{+}\left(\eta_{4}\right) \prod_{j=1}^{2 N} \psi\left(z_{j}\right)\right\rangle
$$




$$
|1\rangle_{+} \underset{\eta_{1}}{\stackrel{\sim}{\rightarrow} \eta_{2}} \sqrt{\frac{\eta_{12}}{2}}\left\langle\psi\left(\eta_{2}\right) \sigma_{-}\left(\eta_{3}\right) \sigma_{+}\left(\eta_{4}\right) \prod_{j=1}^{2 N} \psi\left(z_{j}\right)\right\rangle .
$$

Executing the braiding by the analytic continuation $\eta_{12} \rightarrow \mathrm{e}^{i \pi} \eta_{12}$ gives

$$
\begin{aligned}
|0\rangle_{+} \underset{\eta_{1} \rightarrow \eta_{2}}{\stackrel{\sim}{\sim}}\left\langle\sigma_{+}\left(\eta_{3}\right) \sigma_{+}\left(\eta_{4}\right) \prod_{j=1}^{2 N} \psi\left(z_{j}\right)\right\rangle \stackrel{B_{1}}{\rightarrow}\left\langle\sigma_{+}\left(\eta_{3}\right) \sigma_{+}\left(\eta_{4}\right) \prod_{j=1}^{2 N} \psi\left(z_{j}\right)\right\rangle=|0\rangle_{+}, \\
\left.|1\rangle_{+} \underset{\eta_{1}}{\stackrel{\sim}{\rightarrow} \eta_{2}} \sqrt{\frac{\eta_{12}}{2}}\left\langle\psi\left(\eta_{2}\right) \sigma_{+}\left(\eta_{3}\right) \sigma_{+}\left(\eta_{4}\right) \prod_{j=1}^{2 N} \psi\left(z_{j}\right)\right\rangle\right)_{7} \\
\quad \rightarrow \quad \sqrt{\frac{\mathrm{e}^{i \pi} \eta_{12}}{2}}\left\langle\psi\left(\eta_{2}\right) \sigma_{+}\left(\eta_{3}\right) \sigma_{+}\left(\eta_{4}\right) \prod_{j=1}^{2 N} \psi\left(z_{j}\right)\right\rangle=i|1\rangle_{+}
\end{aligned}
$$

so that the first braid generator in the positive-parity representation is simply

$$
B_{1}^{(4,+)}=\left[\begin{array}{ll}
1 & 0 \\
0 & i
\end{array}\right]
$$

Similarly, to compute $B_{3}^{(4,+)}$ we first fuse $\eta_{3} \rightarrow \eta_{4}$, using the fusion rules (14) and (15) to obtain the short-distance approximation to the computational states, and then braid $\eta_{34} \rightarrow$ $\mathrm{e}^{i \pi} \eta_{34}$ to get, completely in the same way (only replacing $\eta_{12}$ with $\left.\eta_{34}\right), B_{3}^{(4,+)}=B_{1}^{(4,+)}$.

\subsection{Negative-parity representation:}

In order to write explicitly the computational basis in the negative-parity representation we could introduce one extra Majorana fermion to the right of all $\sigma$ fields, still having even number $2 \mathrm{~N}$ of other Majorana fermions. Thus we define the computational basis for 4 Ising anyons in the negative-parity representation

$$
\begin{aligned}
|0\rangle_{-} & \equiv\left\langle\sigma_{+}\left(\eta_{1}\right) \sigma_{+}\left(\eta_{2}\right) \sigma_{+}\left(\eta_{3}\right) \sigma_{-}\left(\eta_{4}\right) \psi\left(z_{0}\right) \prod_{j=1}^{2 N} \psi\left(z_{j}\right)\right\rangle \\
|1\rangle_{-} & \equiv\left\langle\sigma_{+}\left(\eta_{1}\right) \sigma_{-}\left(\eta_{2}\right) \sigma_{+}\left(\eta_{3}\right) \sigma_{+}\left(\eta_{4}\right) \psi\left(z_{0}\right) \prod_{j=1}^{2 N} \psi\left(z_{j}\right)\right\rangle .
\end{aligned}
$$

Again quantum information is encoded in the topological charge $\kappa$ of the first pair of $\sigma$ fields according to $|0\rangle \leftrightarrow \sigma_{+} \sigma_{+},|1\rangle \leftrightarrow \sigma_{+} \sigma_{-}$, however this time the second pair which fixes the total fermion parity of the correlation functions in 19 , has opposite parity compared to that in [16], see Refs. [21, 6] for more detail.

Notice that in general we can insert the extra Majorana fermion between any two pairs of anyons. This will define a new basis of computational states in the negative-parity representation which is related to 197, in which the extra Majorana fermion is to the right of all $\sigma$ fields, by a braid transformation that is diagonal, with elements \pm 1 on the diagonal because the Majorana fermion either commutes or anticommutes with any pair of $\sigma$ fields.

Now we can compute $B_{1}^{(4,-)}$ by first fusing $\eta_{1} \rightarrow \eta_{2}$ and then taking $\eta_{12} \rightarrow \mathrm{e}^{i \pi} \eta_{12}$. Because the short-distance expansions of $\sigma_{+}\left(\eta_{1}\right) \sigma_{ \pm}\left(\eta_{2}\right)$ in the NS sector is independent of the parity of the other fields in the correlator this braid matrix is the same as for the positive-parity representation, i.e., $B_{1}^{(4,-)}=B_{1}^{(4,+)}$ (as matrices because they act on different computational bases). 
In order to compute $B_{3}^{(4,-)}$ we first fuse $\eta_{3} \rightarrow \eta_{4}$, using the fusion rules (14) and (15) to obtain the short-distance approximation to the computational states, which gives

$$
\begin{aligned}
& |0\rangle_{-} \underset{\eta_{3}}{\stackrel{\sim}{\rightarrow} \eta_{4}} \sqrt{\frac{\eta_{34}}{2}}\left\langle\sigma_{+}\left(\eta_{1}\right) \sigma_{+}\left(\eta_{2}\right) \psi\left(\eta_{4}\right) \psi\left(z_{0}\right) \prod_{j=1}^{2 N} \psi\left(z_{j}\right)\right\rangle, \\
& |1\rangle_{-} \underset{\eta_{3} \rightarrow \eta_{4}}{\simeq}\left\langle\sigma_{+}\left(\eta_{1}\right) \sigma_{-}\left(\eta_{2}\right) \psi\left(z_{0}\right) \prod_{j=1}^{2 N} \psi\left(z_{j}\right)\right\rangle .
\end{aligned}
$$

Executing the braiding $\eta_{34} \rightarrow \mathrm{e}^{i \pi} \eta_{34}$ now gives $|0\rangle_{-} \rightarrow \mathrm{e}^{i \pi / 2}|0\rangle_{-}$while $|1\rangle_{-} \rightarrow|1\rangle_{-}$so that the braid generator for the exchange of the last two $\sigma$ fields in the negative-parity representation is

$$
B_{3}^{(4,-)}=\left[\begin{array}{cc}
i & 0 \\
0 & 1
\end{array}\right]
$$

\section{Non-diagonal exchange matrices $B_{2}^{(4, \pm)}$ : Ising fusion rules in the Ramond sector}

Just as in the previous sections we are going to use the fact that the braid matrices for coordinate exchanges of two anyons are independent of the distance between them so we can simplify the computation by allowing the two anyons to fuse, i.e., letting $\eta_{2} \rightarrow \eta_{3}$ in this case, and reading the exchange phases from the analytic continuation of the singular factors containing $\eta_{23}$. However, when we exchange the quasiholes with coordinates $\eta_{2}$ and $\eta_{3}$ there is one extra $\sigma$ field to the right of the quasiholes being exchanged. Therefore, in order to fuse the quasiholes at $\eta_{2}$ and $\eta_{3}$ before braiding them we need to use the OPE of two $\sigma$ fields in the Ramond sector of the Ising model [15], 17].

The OPE of two $\sigma$ fields in the Ramond sector of the Ising model is more complicated than Eq. (14) because the chiral fermion parity in the R-sector is spontaneously broken [16] and therefore that OPE might contain more terms. Fortunately this OPE has been explicitly derived in Ref. [15] (see Eq. (6.47) there, in which we identify $\sigma_{e}=: \varphi_{e}$, where $e= \pm$ is the fermion parity) from the knowledge of the 4-point function computed in Sect. 6 there and could be written as follows

$$
\begin{aligned}
\sigma_{e_{1}}\left(z_{1}\right) \sigma_{e_{2}}\left(z_{2}\right)|e\rangle & =\frac{1}{\sqrt{2} z_{12}^{1 / 8}}\left\{\delta_{e_{1}, e_{2}}|e\rangle+\delta_{e_{1},-e_{2}}|-e\rangle\right. \\
& \left.+\left(e . e_{2}\right) \sqrt{\frac{z_{12}}{2}} \psi\left(\sqrt{z_{1} \cdot z_{2}}\right)\left(\delta_{e_{1}, e_{2}}|-e\rangle+\delta_{e_{1},-e_{2}}|e\rangle\right)\right\}+\cdot \cdot
\end{aligned}
$$

Recall that in the notation of Ref. [15] the ket-vector $|e\rangle$ is defined as the lowest-weight state in the R sector with fermion parity $e$, i.e., $|e\rangle:=\sigma_{e}(0)|0\rangle$. We shall use Eq. 21] in the next Subsections to derive the short-distance approximation of the computational state's wave functions in the bases $\left\{|0\rangle_{+},|1\rangle_{+}\right\}$and $\left\{|0\rangle_{-},|1\rangle_{-}\right\}$.

\subsection{Short-distance OPE of the computational basis wave functions}

In order to simplify the analysis of the fusion process $\eta_{2} \rightarrow \eta_{3}$ we shall denote the R-sector states entering the 4-qh wave functions (16) as

$$
| \pm\rangle:=\sigma_{ \pm}\left(\eta_{4}\right) \prod_{j=1}^{2 N} \psi\left(z_{j}\right)|0\rangle,
$$


and recall that by construction the number $2 N$ of Majorana fermions is even. Let us now apply the R-sector's OPE [21], for $e=+$, to obtain the short-distance expansion of the 4-anyon computational basis vector $|0\rangle_{+}$(in the positive-parity representation) defined in Eq. (16)

$$
\sigma_{+}\left(\eta_{2}\right) \sigma_{+}\left(\eta_{3}\right)|+\rangle \underset{\eta_{2} \rightarrow \eta_{3}}{\simeq} \frac{1}{\sqrt{2} \eta_{23}^{1 / 8}}\left\{|+\rangle+(+.+) \sqrt{\frac{\eta_{23}}{2}} \psi\left(\sqrt{\eta_{2} \eta_{3}}\right)|-\rangle\right\} .
$$

Then, multiplying from the left by $\langle 0| \sigma_{+}\left(\eta_{1}\right)$ we obtain

$$
|0\rangle_{+} \underset{\eta_{2}}{\stackrel{\sim}{\rightarrow} \eta_{3}} \frac{1}{\sqrt{2} \eta_{23}^{1 / 8}}\left\{\left\langle 0\left|\sigma_{+}\left(\eta_{1}\right)\right|+\right\rangle+\sqrt{\frac{\eta_{23}}{2}}\left\langle 0\left|\sigma_{+}\left(\eta_{1}\right)\right|-\right\rangle\right\} .
$$

Notice that the overall phase factor $\eta_{23}^{-1 / 8}$ in the above OPE is exactly canceled by the additional inverse factor coming from the OPE of the Abelian part of the Ising anyons, i.e., from Eq. (15) for $z_{1}=\eta_{2}$ and $z_{2}=\eta_{3}$, and we shall remove it from all expressions below. Thus, recovering the detailed notation, we get the OPE of the first computational-basis state to be

$$
\begin{aligned}
& |0\rangle_{+} \underset{\eta_{2}}{\stackrel{\sim}{\rightarrow} \eta_{3}} \frac{1}{\sqrt{2}}\left\{\left\langle 0\left|\sigma_{+}\left(\eta_{1}\right) \sigma_{+}\left(\eta_{4}\right) \prod_{j=1}^{2 N} \psi\left(z_{j}\right)\right| 0\right\rangle\right. \\
& \left.\quad+\quad \sqrt{\frac{\eta_{23}}{2}}\left\langle 0\left|\sigma_{+}\left(\eta_{1}\right) \psi\left(\sqrt{\eta_{2} \eta_{3}}\right) \sigma_{-}\left(\eta_{4}\right) \prod_{j=1}^{2 N} \psi\left(z_{j}\right)\right| 0\right\rangle\right\} .
\end{aligned}
$$

Similarly, for the computational-basis state $|1\rangle_{+}$we obtain the short-distance expansion

$$
\begin{aligned}
|1\rangle_{+} \underset{\eta_{2}}{\stackrel{\sim}{\rightarrow}} \frac{1}{\eta_{3}} \frac{1}{\sqrt{2}}\left\{\left\langle 0\left|\sigma_{+}\left(\eta_{1}\right) \sigma_{+}\left(\eta_{4}\right) \prod_{j=1}^{2 N} \psi\left(z_{j}\right)\right| 0\right\rangle\right. \\
\left.-\quad \sqrt{\frac{\eta_{23}}{2}}\left\langle 0\left|\sigma_{+}\left(\eta_{1}\right) \psi\left(\sqrt{\eta_{2} \eta_{3}}\right) \sigma_{-}\left(\eta_{4}\right) \prod_{j=1}^{2 N} \psi\left(z_{j}\right)\right| 0\right\rangle\right\} .
\end{aligned}
$$

Adding and subtracting Eqs. (23) and (24) we obtain

$$
\begin{aligned}
& \frac{|0\rangle_{+}+|1\rangle_{+}}{2} \underset{\eta_{2}}{\stackrel{\sim}{\rightarrow} \eta_{3}} \frac{1}{\sqrt{2}}\left\langle 0\left|\sigma_{+}\left(\eta_{1}\right) \sigma_{+}\left(\eta_{4}\right) \prod_{j=1}^{2 N} \psi\left(z_{j}\right)\right| 0\right\rangle \\
& \frac{|0\rangle_{+}-|1\rangle_{+}}{2} \underset{\eta_{2}}{\stackrel{\sim}{\rightarrow} \eta_{3}} \frac{1}{\sqrt{2}} \sqrt{\frac{\eta_{23}}{2}}\left\langle 0\left|\sigma_{+}\left(\eta_{1}\right) \psi\left(\sqrt{\eta_{2} \eta_{3}}\right) \sigma_{-}\left(\eta_{4}\right) \prod_{j=1}^{2 N} \psi\left(z_{j}\right)\right| 0\right\rangle .
\end{aligned}
$$

Eq. 25) will be our starting point for the derivation of the braid matrices in the next subsection because it expresses the correlation functions on the RHS in terms of the computational basis in the LHS in the short-distance limit.

\subsection{Braiding $\eta_{2}$ with $\eta_{3}$ in the positive-parity representation}

The braiding transformation $B_{2}^{(4,+)}$ is represented by the coordinate exchange

$$
\eta_{2} \rightarrow \eta_{3}, \quad \eta_{3} \rightarrow \eta_{2}, \quad \text { so that } \quad \eta_{23} \rightarrow \mathrm{e}^{i \pi} \eta_{23} .
$$

Applying the coordinate exchange over $|0\rangle_{+}$and making analytic continuation in $\eta_{23}$ we get

$$
\begin{aligned}
B_{2}^{(4,+)}|0\rangle_{+} & \underset{\eta_{2}}{\stackrel{\sim}{\rightarrow} \eta_{3}} \frac{1}{\sqrt{2}}\left\{\left\langle 0\left|\sigma_{+}\left(\eta_{1}\right) \sigma_{+}\left(\eta_{4}\right) \prod_{j=1}^{2 N} \psi\left(z_{j}\right)\right| 0\right\rangle\right. \\
+ & \left.\sqrt{\frac{\mathrm{e}^{i \pi} \eta_{23}}{2}}\left\langle 0\left|\sigma_{+}\left(\eta_{1}\right) \psi\left(\sqrt{\eta_{2} \eta_{3}}\right) \sigma_{-}\left(\eta_{4}\right) \prod_{j=1}^{2 N} \psi\left(z_{j}\right)\right| 0\right\rangle\right\} .
\end{aligned}
$$


Now, using $\sqrt{\mathrm{e}^{i \pi}}=i$, we can substitute the correlation functions appearing in the RHS of Eq. (26) with the expressions in the LHS of Eq. (25) to get

$$
B_{2}^{(4,+)}|0\rangle_{+} \underset{\eta_{2}}{\stackrel{\sim}{\rightarrow} \eta_{3}} \frac{|0\rangle_{+}+|1\rangle_{+}}{2}+i \frac{|0\rangle_{+}-|1\rangle_{+}}{2} .
$$

Repeating the same procedure for the computational-basis state $|1\rangle_{+}$we obtain from Eq. 24]

$$
B_{2}^{(4,+)}|1\rangle_{+} \underset{\eta_{2} \rightarrow \eta_{3}}{\simeq} \frac{|0\rangle_{+}+|1\rangle_{+}}{2}-i \frac{|0\rangle_{+}-|1\rangle_{+}}{2}
$$

so that the braid matrix in the basis $\left\{|0\rangle_{+},|1\rangle_{+}\right\}$is

$$
B_{2}^{(4,+)}=\frac{1}{2}\left[\begin{array}{cc}
1+i & 1-i \\
1-i & 1+i
\end{array}\right]=\frac{\mathrm{e}^{i \frac{\pi}{4}}}{\sqrt{2}}\left[\begin{array}{cc}
1 & -i \\
-i & 1
\end{array}\right]
$$

The braid matrix obtained in Eq. (27) completely coincides with the one obtained in Ref. [5] for $\left|\eta_{12} \eta_{34} / \eta_{13} \eta_{24}\right|<1$, where it was denoted as $R_{23}^{(4)}$.

\subsection{Braiding $\eta_{2}$ with $\eta_{3}$ in the negative-parity representation}

Again the computational basis in the negative-parity representation is given by Eq. (19) where $2 N$ is even. Let us now denote

$$
\sigma_{ \pm}\left(\eta_{4}\right) \psi\left(z_{0}\right) \prod_{j=1}^{2 N} \psi\left(z_{j}\right)|0\rangle=:|\mp\rangle
$$

and apply the OPE (21) to obtain the short-distance version of $|0\rangle_{-}$, i.e.,

$$
\sigma_{+}\left(\eta_{2}\right) \sigma_{+}\left(\eta_{3}\right)|+\rangle=\frac{1}{\sqrt{2}}\left\{|+\rangle+\sqrt{\frac{\eta_{23}}{2}} \psi\left(\sqrt{\eta_{2} \eta_{3}}\right)|-\rangle\right\}
$$

and similarly, for the computational-basis state $|1\rangle_{-}$we can use the OPE (21) in the form

$$
\sigma_{-}\left(\eta_{2}\right) \sigma_{+}\left(\eta_{3}\right)|-\rangle=\frac{1}{\sqrt{2}}\left\{|+\rangle-\sqrt{\frac{\eta_{23}}{2}} \psi\left(\sqrt{\eta_{2} \eta_{3}}\right)|-\rangle\right\}
$$

to obtain (after adding and subtracting the results for the two short-distance approximations)

$$
\begin{aligned}
& \frac{|0\rangle_{-}+|1\rangle_{-}}{2} \underset{\eta_{2}}{\stackrel{\sim}{\rightarrow} \eta_{3}} \frac{1}{\sqrt{2}}\left\langle 0\left|\sigma_{+}\left(\eta_{1}\right) \sigma_{+}\left(\eta_{4}\right) \psi\left(z_{0}\right) \prod_{j=1}^{2 N} \psi\left(z_{j}\right)\right| 0\right\rangle \\
& \frac{|0\rangle_{-}-|1\rangle_{-}}{2} \underset{\eta_{2}}{\stackrel{\sim}{\rightarrow} \eta_{3}} \frac{1}{\sqrt{2}} \sqrt{\frac{\eta_{23}}{2}}\left\langle 0\left|\sigma_{+}\left(\eta_{1}\right) \psi\left(\sqrt{\eta_{2} \eta_{3}}\right) \sigma_{-}\left(\eta_{4}\right) \psi\left(z_{0}\right) \prod_{j=1}^{2 N} \psi\left(z_{j}\right)\right| 0\right\rangle .
\end{aligned}
$$

Because the above equation has the same $\eta_{23}$-short-distance structure like Eq. (25), executing the braiding $\eta_{23} \rightarrow \mathrm{e}^{i \pi} \eta_{23}$ produces the same matrix $B_{2}^{(4,-)}=B_{2}^{(4,+)}$ (as matrices). Thus we conclude that $B_{2}^{(4,-)}$ is indeed given by Eq. (13).

\section{Braid generators for exchanges of $2 n+2$ Ising anyons: wave-function approach}

To summarize our results for the exchanges of 4 Ising anyons, we note that the generators of the negative-parity representation (13) of $\mathscr{B}_{4}$ completely coincide with those obtained directly from the $\gamma$ matrices for $\mathrm{SO}(4)$ in Sect. III of Ref. [21], i.e.,

$$
B_{j}^{(4,-)}=R_{j}^{(2,-)}, \quad j=1,2,3 \text {. }
$$


In contrast, the second generator of the positive-parity representation of $\mathscr{B}_{4}$ is different from that obtained in the $\gamma$ matrix approach. Nevertheless, it is easy to see that the positive-parity representation (12), obtained from the 4-quasiholes Pfaffian wave functions, is completely equivalent to the positive-parity representation in the $\gamma$-matrix approach $R_{j}^{(2,+)}$ (see Eqs. (9) and (10) in Ref. [21]), i.e.,

$$
B_{j}^{(4,+)}=Z R_{j}^{(2,+)} Z, \quad j=1,2,3, \quad Z=\left(B_{1}^{(4, \pm)}\right)^{2}=\left[\begin{array}{cc}
1 & 0 \\
0 & -1
\end{array}\right],
$$

and the equivalence transformation is explicitly given by the Pauli matrix $Z$. This is so because the $Z$ matrix commutes with the diagonal $B_{1}^{(4,+)}$ and $B_{3}^{(4,+)}$, however, changes the signs of the off-diagonal elements of $B_{2}^{(4,+)}$. It can also be directly seen that the two representations (12) and (13) of $\mathscr{B}_{4}$ are inequivalent, see e.g., Sect. III in Ref. [21]. Thus we conclude that the representations (12) and (13) of $\mathscr{B}_{4}$ are equivalent to the spinor representations of $\mathrm{SO}(4)$ with the corresponding parity [7, 21].

In this section, we shall generalize this result to the braid representations for $2 n+2$ Ising anyons. Our strategy to compute the braid matrices $B_{j}^{(2 n+2, \pm)}$, describing the exchanges of $2 n+2$ anyons in the Ising representation of the braid group $\mathscr{B}_{2 n+2}$, would be to fuse some pair of $\sigma$ fields, representing one of the qubits in our $n$ qubit system, which has the effect of projecting out this qubit. The resulting states after fusion will belong to one of the two representations of $\mathscr{B}_{2 n}$ with positive or negative parity so that we can express the braid matrices $B_{j}^{(2 n+2, \pm)}$ recursively in terms of $B_{j}^{(2 n, \pm)}$. More precisely, we shall prove the following recurrence relations:

Proposition 1: the $2^{n} \times 2^{n}$ dimensional matrices $B_{j}^{(2 n+2, \pm)},(1 \leq j \leq 2 n+1)$ representing the generators of the braid group $\mathscr{B}_{2 n+2}$ in the computational bases 36 can be expressed recursively in terms of the braid matrices $B_{j}^{(2 n, \pm)},(1 \leq j \leq 2 n-1)$ generating the Ising representation of $\mathscr{B}_{2 n}$ as follows:

$$
\begin{aligned}
& B_{j}^{(2 n+2,+)}=B_{j}^{(2 n+2,-)} \quad \text { for } \quad 1 \leq j \leq 2 n \\
& B_{j}^{(2 n+2, \pm)}=B_{j}^{(2 n, \pm)} \otimes \mathbb{I}_{2} \quad \text { for } \quad 1 \leq j \leq 2 n-3 \\
& B_{j}^{(2 n+2, \pm)}=B_{j-2}^{(2 n, \pm)} \oplus B_{j-2}^{(2 n, \mp)} \quad \text { for } \quad 3 \leq j \leq 2 n+1
\end{aligned}
$$

Proof: We shall prove this proposition by induction with a base $n=2$. To this end we shall first explicitly prove statements (i)-(iii) for $n=2$. The braid generators $B_{j}^{(6, \pm)}$ are $4 \times 4$ dimensional matrices defined in the computational basis for 6 anyons (corresponding to 2 qubits, encoded in the first two pairs of $\sigma$ fields, plus one extra inert pair, formed by the last two $\sigma$ fields). For positive parity this basis can be written as [6]

$$
\begin{array}{ll}
|00\rangle_{+}=\left\langle\sigma_{+} \sigma_{+} \sigma_{+} \sigma_{+} \sigma_{+} \sigma_{+}\right\rangle, & |01\rangle_{+}=\left\langle\sigma_{+} \sigma_{+} \sigma_{+} \sigma_{-} \sigma_{+} \sigma_{-}\right\rangle, \\
|10\rangle_{+}=\left\langle\sigma_{+} \sigma_{-} \sigma_{+} \sigma_{+} \sigma_{+} \sigma_{-}\right\rangle, & |11\rangle_{+}=\left\langle\sigma_{+} \sigma_{-} \sigma_{+} \sigma_{-} \sigma_{+} \sigma_{+}\right\rangle,
\end{array}
$$

where we skipped for simplicity the product of the even number of Majorana fermions as well as the coordinates $\eta_{j}$ of the fields $\sigma_{ \pm}\left(\eta_{j}\right)$. In order to find the braid matrices $B_{1}^{(6,+)}$ or $B_{2}^{(6,+)}$, exchanging $\eta_{1} \leftrightarrow \eta_{2}$ or $\eta_{2} \leftrightarrow \eta_{3}$, respectively, we can first fuse $\eta_{5} \rightarrow \eta_{6}$. The results after fusion are computational states from the positive- or negative-parity representations of $\mathscr{B}_{4}$, i.e.,

$$
\begin{aligned}
& |00\rangle_{+} \underset{\eta_{5} \rightarrow \eta_{6}}{\rightarrow}\left\langle\sigma_{+} \sigma_{+} \sigma_{+} \sigma_{+}\right\rangle=|0\rangle_{+}, \quad|01\rangle_{+} \underset{\eta_{5} \rightarrow \eta_{6}}{\rightarrow}\left\langle\sigma_{+} \sigma_{+} \sigma_{+} \sigma_{-} \psi\right\rangle=|0\rangle_{-} \\
& |10\rangle_{+} \underset{\eta_{5} \rightarrow \eta_{6}}{\rightarrow}\left\langle\sigma_{+} \sigma_{-} \sigma_{+} \sigma_{+} \psi\right\rangle=|1\rangle_{-}, \quad|11\rangle_{+} \underset{\eta_{5} \rightarrow \eta_{6}}{\rightarrow}\left\langle\sigma_{+} \sigma_{-} \sigma_{+} \sigma_{-}\right\rangle=|1\rangle_{+} .
\end{aligned}
$$


Let us first compute $B_{1}^{(6,+)}$. Using the above fusion results for the computational basis, as well as Eqs. (12) and (13) for $B_{1}^{(4, \pm)}$, we obtain

$$
\begin{aligned}
& B_{1}^{(6,+)}|00\rangle_{+} \simeq B_{1}^{(4,+)}|0\rangle_{+}=|0\rangle_{+} \simeq|00\rangle_{+} \\
& B_{1}^{(6,+)}|01\rangle_{+} \simeq B_{1}^{(4,-)}|0\rangle_{-}=|0\rangle_{-} \simeq|01\rangle_{+} \\
& B_{1}^{(6,+)}|10\rangle_{+} \simeq B_{1}^{(4,+)}|1\rangle_{+}=i|1\rangle_{+} \simeq i|10\rangle_{+} \\
& B_{1}^{(6,+)}|11\rangle_{+} \simeq B_{1}^{(4,-)}|1\rangle_{-}=i|1\rangle_{-} \simeq i|11\rangle_{+},
\end{aligned}
$$

so that $B_{1}^{(6,+)}=\operatorname{diag}(1,1, i, i)=B_{1}^{(4,+)} \otimes \mathbb{I}_{2}$. Next, in the same way we compute $B_{2}^{(6,+)}$ by

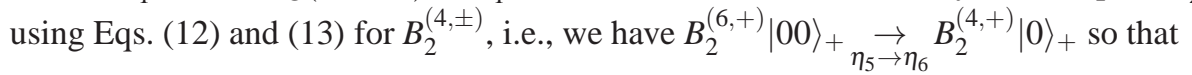

$$
B_{2}^{(6,+)}|00\rangle_{+} \simeq \frac{\mathrm{e}^{i \frac{\pi}{4}}}{\sqrt{2}}\left(|0\rangle_{+}-i|1\rangle_{+}\right) \simeq \frac{\mathrm{e}^{i \frac{\pi}{4}}}{\sqrt{2}}\left(|00\rangle_{+}-i|11\rangle_{+}\right) .
$$

Similarly, we have $B_{2}^{(6,+)}|01\rangle_{+} \underset{\eta_{5} \rightarrow \eta_{6}}{\rightarrow} B_{2}^{(4,-)}|0\rangle_{-}$so that

$$
B_{2}^{(6,+)}|01\rangle_{+} \simeq \frac{\mathrm{e}^{i \frac{\pi}{4}}}{\sqrt{2}}\left(|0\rangle_{-}-i|1\rangle_{-}\right) \simeq \frac{\mathrm{e}^{i \frac{\pi}{4}}}{\sqrt{2}}\left(|01\rangle_{+}-i|10\rangle_{+}\right)
$$

Continuing in this way with the states $|10\rangle_{+}$and $|11\rangle_{+}$we find

$$
B_{2}^{(6,+)}=\frac{\mathrm{e}^{i \frac{\pi}{4}}}{\sqrt{2}}\left[\begin{array}{cccc}
1 & 0 & 0 & -i \\
0 & 1 & -i & 0 \\
0 & -i & 1 & 0 \\
-i & 0 & 0 & 1
\end{array}\right]
$$

Next, in order to compute the rest of the braid generators we can instead fuse the first two Ising anyons. This projects out the first qubit so that the computational basis becomes

$$
\begin{aligned}
& |00\rangle_{+} \underset{\eta_{1} \rightarrow \eta_{2}}{\rightarrow}\left\langle\sigma_{+} \sigma_{+} \sigma_{+} \sigma_{+}\right\rangle=|0\rangle_{+}, \quad|01\rangle_{+} \underset{\eta_{1} \rightarrow \eta_{2}}{\rightarrow}\left\langle\sigma_{+} \sigma_{-} \sigma_{+} \sigma_{-}\right\rangle=|1\rangle_{+} \\
& |10\rangle_{+} \underset{\eta_{1} \rightarrow \eta_{2}}{\rightarrow}\left\langle\psi \sigma_{+} \sigma_{+} \sigma_{+} \sigma_{-}\right\rangle=|0\rangle_{-}, \quad|11\rangle_{+} \underset{\eta_{1} \rightarrow \eta_{2}}{\rightarrow}\left\langle\psi \sigma_{+} \sigma_{-} \sigma_{+} \sigma_{+}\right\rangle=|1\rangle_{-} .
\end{aligned}
$$

Consider, e.g., the braid matrix $B_{3}^{(6,+)}$. It is obvious that

$$
\begin{aligned}
& B_{3}^{(6,+)}|00\rangle_{+} \simeq B_{1}^{(4,+)}|0\rangle_{+}, \quad B_{3}^{(6,+)}|01\rangle_{+} \simeq B_{1}^{(4,+)}|1\rangle_{+}, \\
& B_{3}^{(6,+)}|10\rangle_{+} \simeq B_{1}^{(4,-)}|0\rangle_{-}, \quad B_{3}^{(6,+)}|11\rangle_{+} \simeq B_{1}^{(4,-)}|1\rangle_{-},
\end{aligned}
$$

so that $B_{3}^{(6,+)}=B_{1}^{(4,+)} \oplus B_{1}^{(4,-)}=\mathbb{I}_{2} \otimes B_{1}^{(4,+)}$ because $B_{1}^{(4,-)}=B_{1}^{(4,+)}$. Here we used the sign $\oplus$ to denote the direct sum of matrices. Completely in the same way we find $B_{4}^{(6,+)}=B_{2}^{(4,+)} \oplus B_{2}^{(4,-)}=\mathbb{I}_{2} \otimes B_{2}^{(4,+)}$ because $B_{2}^{(4,-)}=B_{2}^{(4,+)}$ and $B_{5}^{(6,+)}=B_{3}^{(4,+)} \oplus B_{3}^{(4,-)}=$ $\operatorname{diag}(1, i, i, 1)$. Notice that the last generator $B_{5}^{(6,+)}$ is not a tensor product of $\mathbb{I}_{2}$ and $B_{3}^{(4,+)}$ because $B_{3}^{(4,-)} \neq B_{3}^{(4,+)}$.

Next we have to repeat the above computation of the braid generators $B_{j}^{(6,-)}$ in the negative-parity representation. The computational basis is now given by [6]

$$
\begin{array}{ll}
|00\rangle_{-}=\left\langle\sigma_{+} \sigma_{+} \sigma_{+} \sigma_{+} \sigma_{+} \sigma_{-}\right\rangle, & |01\rangle_{-}=\left\langle\sigma_{+} \sigma_{+} \sigma_{+} \sigma_{-} \sigma_{+} \sigma_{+}\right\rangle \\
|10\rangle_{-}=\left\langle\sigma_{+} \sigma_{-} \sigma_{+} \sigma_{+} \sigma_{+} \sigma_{+}\right\rangle, & |11\rangle_{-}=\left\langle\sigma_{+} \sigma_{-} \sigma_{+} \sigma_{-} \sigma_{+} \sigma_{-}\right\rangle
\end{array}
$$


because the total parity of the $\sigma$ fields must be negative (and this is compensated by the odd number of Majorana fermions inside the CFT correlators which are omitted again). It is not difficult to see that the results for $B_{j}^{(6,-)}$ are very similar to those for $B_{j}^{(6,+)}$ just in each step all + are replaced by - and vice versa. For example $B_{1}^{(6,-)}=\operatorname{diag}(1,1, i, i)=B_{1}^{(4,-)} \otimes \mathbb{I}_{2}$, $B_{2}^{(6,-)}=B_{2}^{(6,+)}\left(\right.$ as a consequence of $\left.B_{2}^{(4,-)}=B_{2}^{(4,+)}\right), B_{3}^{(6,-)}=B_{1}^{(4,-)} \oplus B_{1}^{(4,+)}=\mathbb{I}_{2} \otimes B_{1}^{(4,-)}$, etc. The only difference is in the last generator where $B_{5}^{(6,-)}=B_{3}^{(4,-)} \oplus B_{3}^{(4,+)}=\operatorname{diag}(i, 1,1, i)$ cannot be written as a tensor product.

The results for the braid matrices $B_{j}^{(6, \pm)}$ can be summarized as follows: $B_{2}^{(6, \pm)}$ are equal and given by (32) and the others are given explicitly by

$$
\begin{array}{ll}
B_{1}^{(6, \pm)}=B_{1}^{(4, \pm)} \otimes \mathbb{I}_{2}, & B_{3}^{(6, \pm)}=B_{1}^{(4, \pm)} \oplus B_{1}^{(4, \mp)}=\mathbb{I}_{2} \otimes B_{1}^{(4, \pm)}, \\
B_{4}^{(6, \pm)}=\mathbb{I}_{2} \otimes B_{2}^{(4, \pm)}, & B_{5}^{(6, \pm)}=B_{3}^{(4, \pm)} \oplus B_{3}^{(4, \mp)}
\end{array}
$$

where $B_{j}^{(4, \pm)}$ are defined in Eqs. (12) and (13). This proves statements (ii) and (iii) for the case $n=2$, which is our induction base. Now it is easy to see that in addition these braid matrices satisfy

$$
B_{j}^{(6,+)} \equiv B_{j}^{(6,-)} \quad \text { for } \quad 1 \leq j \leq 4,
$$

which proves the statement (i) for the base $n=2$.

It can also be seen that the positive-parity representation of the braid group $\mathscr{B}_{6}$ obtained here is completely equivalent to the one derived earlier in Refs. [19, 5, 22] and the equivalence is established by the braid matrix $U=B_{4}^{(6,+)} B_{3}^{(6,+)} B_{5}^{(6,+)} B_{4}^{(6,+)}$ representing the exchange of the pairs $\left(\eta_{3}, \eta_{4}\right)$ and $\left(\eta_{5}, \eta_{6}\right)$ (recall that in the representation of Refs. [19, 5, 22] the inert pair was $\sigma\left(\eta_{3}\right) \sigma\left(\eta_{4}\right)$ while here the inert pair is $\left.\sigma\left(\eta_{5}\right) \sigma\left(\eta_{6}\right)\right)$.

Induction step: Let us assume that the statements (i)-(iii) are fulfilled for the matrices $B_{j}^{(2 n, \pm)}$. We must first specify the basis of computational states for $2 n+2$ anyons in which the braid matrices are represented. The general scheme for representing $n$ qubits in terms of $2 n+2$ Ising anyons could be described as follows [21, 6]. We group the $2 n+2$ fields $\sigma$ into $n+1$ pairs and encode information into the first $n$ pairs: the state of the $i$-th qubit is $|0\rangle$ if the $i$-th pair of $\sigma$ fields is $\sigma_{+}\left(\eta_{2 i-1}\right) \sigma_{+}\left(\eta_{2 i}\right)$ (i.e., it fuses to the channel of $\mathbb{I}$ ) or $|1\rangle$ if the $i$-th pair is $\sigma_{+}\left(\eta_{2 i-1}\right) \sigma_{-}\left(\eta_{2 i}\right)$ (i.e., it fuses to the channel of $\left.\psi\right)$. The last pair $\sigma_{+}\left(\eta_{2 n+1}\right) \sigma_{c}\left(\eta_{2 n+2}\right)$ contains no information because its state $c$ is determined by the requirement to have a nonzero CFT correlator, i.e., $c=\prod_{i=1}^{2 n} c_{i}$. Thus, the computational states in the positive/negativeparity representation of our $n$-qubit system are defined as CFT correlation functions of the $(2 n+2)$ non-Abelian $\sigma$ fields and an even/odd number $N$ of Majorana fermions

$$
\left|c_{1}, \ldots, c_{n}\right\rangle_{ \pm}=\left\langle\sigma_{+} \sigma_{c_{1}} \cdots \sigma_{+} \sigma_{c_{n}} \sigma_{+} \sigma_{c} \prod_{j=1}^{N} \psi\left(z_{j}\right)\right\rangle .
$$

The parity of the representation is denoted by the subscript of the computational basis states: it is ' + ' for positive parity (corresponding to even number $N$ of Majorana fermions) and '-' for negative parity (corresponding to odd number $N$ of Majorana fermions). In other words, $c_{j}=+$ corresponds to the state $|0\rangle$ of the $j$-th qubit, while $c_{j}=-$ corresponds to the state $|1\rangle$. Following our strategy we can first fuse the fields $\sigma\left(\eta_{2 n-1}\right)$ and $\sigma\left(\eta_{2 n}\right)$ corresponding to the last qubit which has the effect of projecting out the last qubit, i.e.,

$$
\left|c_{1}, c_{2}, \ldots, c_{n-1}, c_{n}\right\rangle_{ \pm} \underset{\eta_{2 n-1} \rightarrow \eta_{2 n}}{\longrightarrow}\left\{\begin{array}{cl}
\left|c_{1}, c_{2}, \ldots, c_{n-1}\right\rangle_{ \pm} & \text {if } c_{n}=+ \\
\pm\left|c_{1}, c_{2}, \ldots, c_{n-1}\right\rangle_{\mp} & \text { if } c_{n}=-
\end{array} .\right.
$$


(The sign \pm in front of the $(n-1)$-qubit computational state for $c_{n}=-$ coincides with the eigenvalue of the braid generator $B_{2 n-1}^{(2 n, \mp)}$, however, it is unimportant for our purposes and we will skip it below.) This means that the computational states after projection will be organized in pairs, such as $|\underbrace{0,1,0 \ldots, 0,1,1}_{n-1}\rangle_{+},|\underbrace{0,1,0 \ldots, 0,1,1}_{n-1}\rangle_{-}$, having exactly the same state of the $(n-1)$-qubit system, however with opposite parity, on which the $(2 n+2)$-particle exchange $B_{j}^{(2 n+1, \pm)}$ would act by $B_{j}^{(2 n,+)}$ and $B_{j}^{(2 n,-)}$. Given that we do not touch the last 4 anyons, corresponding to the last qubit and the inert pair, and provided that the braid matrices acting trivially on the last 4 anyons in the two representations are the same $B_{j}^{(2 n,+)}=B_{j}^{(2 n,-)}$, $(1 \leq j \leq 2 n-2)$ because of the inductive step (i), we arrive at Eq. (29).

On the other hand we can fuse instead the first two $\sigma$ fields corresponding to projecting out the first qubit. Then, in the first half of the computational states, containing $\sigma_{+} \sigma_{+}$as a first pair, the result of fusion is $\mathbb{I}$ so that the remaining CFT correlation function describes a $(n-1)$-qubit computational state with $2 n$ anyons and the same parity. In the second half of the computational states, containing $\sigma_{+} \sigma_{-}$as a first pair, the result of fusion is $\psi$ so that the remaining CFT correlation function describes a $(n-1)$-qubit computational state with $2 n$ anyons however with the opposite parity compared to the original one, i.e.,

$$
\left|c_{1}, c_{2}, \ldots, c_{n-1}, c_{n}\right\rangle_{ \pm} \underset{\eta_{1} \rightarrow \eta_{2}}{\longrightarrow}\left\{\begin{array}{rl}
\left|c_{2}, \ldots, c_{n-1}, c_{n}\right\rangle_{ \pm} & \text {if } c_{1}=+ \\
-\left|c_{2}, \ldots, c_{n-1}, c_{n}\right\rangle_{\mp} & \text { if } c_{1}=-
\end{array} .\right.
$$

The minus sign multiplying the $(n-1)$-qubit state when $c_{1}=-1$, which is totally unimportant here because the braid generators which we want to compute act linearly, comes from the fact that after fusing the first two anyons in this case we get one Majorana fermion on the left of all remaining $\sigma$ fields, which we have to move, according to our convention, all the way to the right of them as it is in the definition (36) of the computational states with negative parity. This produces one minus sign for each pair of $\sigma$ 's which is in the state $|1\rangle$ but the total sign for this move is always '-' . Next, executing exchanges on the remaining $2 n$ anyons, that do not touch the first qubit, we immediately find the recurrence relations (30) (notice the shift $j \rightarrow j-2$ in the indices of the braid matrices due to renaming of the remaining anyons coordinates $\eta_{j}^{\prime}=$ $\eta_{j-2}$ for $3 \leq j \leq 2 n+2$ ). The above mentioned extra minus sign does not change anything because the braidings act linearly. To illustrate this consider, e.g., the action of $B_{6}^{(8,+)}$ on the states $|000\rangle_{+}$and $|100\rangle_{+}$, which after projecting the first qubit will go to $\pm|00\rangle_{ \pm}$. We have for the first state $B_{6}^{(8,+)}|000\rangle_{+} \simeq B_{4}^{(6,+)}\left(|00\rangle_{+}\right)=\frac{\mathrm{e}^{i \frac{\pi}{4}}}{\sqrt{2}}\left(|00\rangle_{+}-i|01\rangle_{+}\right)=\frac{\mathrm{e}^{i \frac{\pi}{4}}}{\sqrt{2}}\left(|000\rangle_{+}-i|001\rangle_{+}\right)$, while for the second one

$$
\begin{aligned}
& B_{6}^{(8,+)}|100\rangle_{+} \underset{\eta_{1} \rightarrow \eta_{2}}{\longrightarrow} B_{4}^{(6,-)}\left(-|00\rangle_{-}\right)=-\frac{\mathrm{e}^{i \frac{\pi}{4}}}{\sqrt{2}}\left(|00\rangle_{-}-i|01\rangle_{-}\right) \underset{\eta_{1} \rightarrow \eta_{2}}{\simeq} \\
& -\frac{\mathrm{e}^{i \frac{\pi}{4}}}{\sqrt{2}}\left(-|100\rangle_{+}+i|101\rangle_{+}\right)=\frac{\mathrm{e}^{i \frac{\pi}{4}}}{\sqrt{2}}\left(|100\rangle_{+}-i|101\rangle_{+}\right) .
\end{aligned}
$$

Finally we must prove statement (28) for the matrices $B_{j}^{(2 n+2, \pm)}$. Indeed, we have assumed that $B_{j}^{(2 n,-)}=B_{j}^{(2 n,+)}$ for $1 \leq j \leq 2 n-2$, which is the inductive step (i) for $B_{j}^{(2 n, \pm)}$. Then we can first consider the case when $1 \leq j \leq 2 n-3$ and use 29 ) to find

$$
B_{j}^{(2 n+2,-)}=B_{j}^{(2 n,-)} \otimes \mathbb{I}_{2}=B_{j}^{(2 n,+)} \otimes \mathbb{I}_{2}=B_{j}^{(2 n+2,+)}, 1 \leq j \leq 2 n-3 .
$$

For the rest of the braid matrices we can use (iii) to prove that for $3 \leq j \leq 2 n$

$$
B_{j}^{(2 n+2,-)}=B_{j-2}^{(2 n,-)} \oplus B_{j-2}^{(2 n,+)}=B_{j-2}^{(2 n,+)} \oplus B_{j-2}^{(2 n,-)}=B_{j}^{(2 n+2,+)},
$$


because $B_{j^{\prime}}^{(2 n,+)}=B_{j^{\prime}}^{(2 n,+)}$ for $1 \leq j^{\prime} \leq 2 n-2$ where $j^{\prime}=j-2$. This completes the proof of the Proposition.

Corollary: The recurrence relations (i)-(iii) in the Proposition allow for the following explicit representation for most of the braid matrices $B_{j}^{(2 n+2, \pm)}$, which might be useful:

$$
\begin{aligned}
B_{2 j-1}^{(2 n+2, \pm)} & =\underbrace{\mathbb{I}_{2} \otimes \cdots \otimes \mathbb{I}_{2}}_{j-1} \otimes\left[\begin{array}{cc}
1 & 0 \\
0 & i
\end{array}\right] \otimes \underbrace{\mathbb{I}_{2} \otimes \cdots \otimes \mathbb{I}_{2}}_{n-j}, \text { for } 1 \leq j \leq n, \\
B_{2 j}^{(2 n+2, \pm)} & =\underbrace{\mathbb{I}_{2} \otimes \cdots \otimes \mathbb{I}_{2}}_{j-1} \otimes \frac{\mathrm{e}^{i \frac{\pi}{4}}}{\sqrt{2}}\left[\begin{array}{cccc}
1 & 0 & 0 & -i \\
0 & 1 & -i & 0 \\
0 & -i & 1 & 0 \\
-i & 0 & 0 & 1
\end{array}\right] \otimes \underbrace{\mathbb{I}_{2} \otimes \cdots \otimes \mathbb{I}_{2}}_{n-j-1}
\end{aligned}
$$

for $n \geq 2$ and $1 \leq j \leq n-1$, as well as

$$
B_{2 n}^{(2 n+2, \pm)}=\underbrace{\mathbb{I}_{2} \otimes \cdots \otimes \mathbb{I}_{2}}_{n-1} \otimes \frac{\mathrm{e}^{i \frac{\pi}{4}}}{\sqrt{2}}\left[\begin{array}{cc}
1 & -i \\
-i & 1
\end{array}\right]
$$

plus only one more (non-trivial) recursive relation for the last (diagonal) generator

$$
B_{2 n+1}^{(2 n+2, \pm)}=B_{2 n-1}^{(2 n, \pm)} \oplus B_{2 n-1}^{(2 n, \mp)}
$$

with a base $B_{3}^{(4, \pm)}$, given in Eqs. (12) and (13). Notice that braid matrices 40) are different in the two representations with even or odd number of Majorana fermions and cannot be expressed as tensor products of braid operators form the braid groups, such as $\mathscr{B}_{2 n}$ or $\mathscr{B}_{4}$, for smaller number of anyons. To illustrate the derivation of the explicit formulas (37), (38) and (39) consider for example the matrix $B_{4}^{(2 n+2, \pm)}$ (i.e., $j=2$ in Eq. (38)): on the one hand we have from Eq. 29.

$$
B_{4}^{(2 n+2, \pm)}=B_{4}^{(2 n, \pm)} \otimes \mathbb{I}_{2}=\cdots=B_{4}^{(8, \pm)} \otimes \mathbb{I}_{2^{n-3}},
$$

where we have used that we can add tensor factors of $\mathbb{I}_{2}$ to the right, reducing at the same time the value of $2 n$, until $4 \leq 2 n-3$, i.e., until $2 n \geq 8$, which gives rise to $(2 n-8) / 2+1$ factors and on the other hand, using Eq. [30, we have

$$
B_{4}^{(2 n+2, \pm)}=B_{4}^{(8, \pm)} \otimes \mathbb{I}_{2^{n-3}}=\mathbb{I}_{2} \otimes B_{2}^{(6, \pm)} \otimes \mathbb{I}_{2^{n-3}},
$$

because $B_{4}^{(8, \pm)}=B_{2}^{(6, \pm)} \oplus B_{2}^{(6, \mp)}=\mathbb{I}_{2} \otimes B_{2}^{(6, \pm)}$ (note that $B_{2}^{(6,+)}=B_{2}^{(6,-)}$ ). Similarly, combining Eqs. (30) for $j=2 n$ and (28) we can verify Eq. (39)

$$
B_{2 n}^{(2 n+2, \pm)}=B_{2 n-2}^{(2 n, \pm)} \oplus B_{2 n-2}^{(2 n+2, \mp)}=\mathbb{I}_{2} \otimes B_{2 n-2}^{(2 n, \pm)}=\mathbb{I}_{2^{n-1}} \otimes B_{2}^{(4, \pm)} .
$$

Equations (37), 38), 39) and (40) give the most explicit expressions for the generators $B_{j}^{(2 n+2, \pm)}$ of the braid group $\mathscr{B}_{2 n+2}$ in the two Ising-model representations with opposite parity. These equations also allow us to express the braid matrices, representing the exchanges of Ising anyons in the multi-anyon Pfaffian wave functions, in terms of the universal $R$ matrix for the Ising model [8, 22] (or, equivalently, for the $\widehat{s u(2)}$, Wess-Zumino-Witten model). Note the crucial role of the projectors to states with definite parity leading to topological entanglement [22], i.e., to the fact that not all braid generators are expressible as tensor products of braid matrices with smaller dimensions and the unit matrix $\mathbb{I}_{2}$. 


\section{Proof of the Nayak-Wilczek conjecture}

Proposition 2: The representations of the braid group $\mathscr{B}_{2 n+2}$ with positive or negative fermion parity, constructed from the multi-anyon Pfaffian wave functions, are equivalent to the spinor representations, with the corresponding parity, of $\mathscr{B}_{2 n+2}$ constructed from the spinor representations of $\mathrm{SO}(2 n+2)$. In more detail, the generators $B_{j}^{(2 n+2, \pm)}$ of $\mathscr{B}_{2 n+2}$ in the wavefunction representations can be expressed in terms of the generators $R_{j}^{(n+1, \pm)}$ in the $\mathrm{SO}(2 n+2)$ representations as follows

$$
B_{j}^{(2 n+2, \pm)}=\left(C^{(2 n+2, \pm)}\right)^{-1} R_{j}^{(n+1, \pm)} C^{(2 n+2, \pm)}, \quad 1 \leq j \leq 2 n+1,
$$

where the equivalence matrices for the positive/negative parity $(+/-)$ are given explicitly by the product of all diagonal generators

$$
C^{(2 n+2, \pm)}=\prod_{j=1}^{n+1} R_{2 j-1}^{(n+1, \pm)}=\prod_{j=1}^{n+1} B_{2 j-1}^{(2 n+2, \pm)} .
$$

Proof: First of all it is easy to see that the diagonal generators in the wave-function and spinor representations of $\mathscr{B}_{2 n+2}$ coincide, i.e.,

$$
B_{2 j-1}^{(2 n+2, \pm)}=R_{2 j-1}^{(n+1, \pm)}, \quad 1 \leq j \leq n+1
$$

Indeed, as we can see from [21], the diagonal matrices $B_{2 j-1}^{(2 n+2, \pm)}$ with indices $1 \leq 2 j-1 \leq$ $2 n-1$, given explicitly in Eq. (37) above, are completely identical to the diagonal matrices $R_{2 j-1}^{(n+1, \pm)}$ for $1 \leq 2 j-1 \leq 2 n-1$, given explicitly in Eq. (26) in Ref. [21]. In addition, the last diagonal matrices are equal because they satisfy the same recurrence relations

$$
B_{2 n+1}^{(2 n+2, \pm)}=B_{2 n-1}^{(2 n, \pm)} \oplus B_{2 n-1}^{(2 n, \mp)} \quad \text { or } \quad R_{2 n+1}^{(n+1, \pm)}=R_{2 n-1}^{(n, \pm)} \oplus R_{2 n-1}^{(n, \mp)}
$$

with exactly the same bases, $B_{3}^{(4, \pm)}=R_{3}^{(2, \pm)}$. Because the equivalence matrices (42) are diagonal by construction, the matrices $B_{2 j-1}^{(2 n+2, \pm)}$ and $R_{2 j-1}^{(n+1, \pm)}$ trivially satisfy Eq. 441, and therefore we only need to consider the non-diagonal matrices. The non-diagonal matrices in the $\mathrm{SO}(2 n+2)$ representation $R_{2 j}^{(n+1, \pm)}=P_{ \pm}^{(n+1)} R_{2 j}^{(n+1)} P_{ \pm}^{(n+1)}$ can be expressed [21] as projections of the unprojected matrices ( $\sigma_{1}$ and $\sigma_{2}$ below denote the Pauli matrices)

$$
R_{2 j}^{(n+1)}=\underbrace{\mathbb{I}_{2} \otimes \cdots \otimes \mathbb{I}_{2}}_{j-1} \otimes \frac{\mathrm{e}^{i \frac{\pi}{4}}}{\sqrt{2}}\left(\mathbb{I}_{4}-i \sigma_{2} \otimes \sigma_{2}\right) \otimes \underbrace{\mathbb{I}_{2} \otimes \cdots \otimes \mathbb{I}_{2}}_{n-j} .
$$

On the other hand, Eqs. (38) and (39) suggest that the braid generators $B_{2 j}^{(2 n+2, \pm)}$ can be expressed in a similar way as projections $B_{2 j}^{(2 n+2, \pm)}=P_{ \pm}^{(n+1)} B_{2 j}^{(2 n+2)} P_{ \pm}^{(n+1)}$ (with the same projectors as for $R_{2 j}^{(n+1, \pm)}$ given in Ref. [21]), of the unprojected matrices

$$
B_{2 j}^{(2 n+2)}=\underbrace{\mathbb{I}_{2} \otimes \cdots \otimes \mathbb{I}_{2}}_{j-1} \otimes \frac{\mathrm{e}^{i \frac{\pi}{4}}}{\sqrt{2}}\left(\mathbb{I}_{4}-i \sigma_{1} \otimes \sigma_{1}\right) \otimes \underbrace{\mathbb{I}_{2} \otimes \cdots \otimes \mathbb{I}_{2}}_{n-j},
$$

i.e., if we define the unprojected matrices $B_{2 j}^{(2 n+2)}$ as in Eq. (43) and apply the projectors $P_{ \pm}^{(n+1)}$ as described in [21] then the projected matrices will completely coincide with $B_{2 j}^{(2 n+2, \pm)}$ as given in Eqs. (38) and (39). Next, we can directly prove that the unprojected matrices are related by

$$
B_{2 j}^{(2 n+2)}=\left(C^{(2 n+2)}\right)^{-1} R_{2 j}^{(n+1)} C^{(2 n+2)}, \quad 2 \leq 2 j \leq 2 n,
$$


where the unprojected conjugation matrix is

$$
C^{(2 n+2)}=\underbrace{S \otimes \cdots \otimes S}_{n+1}=\prod_{j=1}^{n+1} R_{2 j-1}^{(n+1)}, \quad S=\left[\begin{array}{ll}
1 & 0 \\
0 & i
\end{array}\right] .
$$

This is simply because of the identity

$$
S^{-1} \sigma_{2} S=\sigma_{1} \quad \Rightarrow \quad(S \otimes S)^{-1}\left(\sigma_{2} \otimes \sigma_{2}\right)(S \otimes S)=\sigma_{1} \otimes \sigma_{1} .
$$

Now, projecting both sides of (44) with the projectors $P_{ \pm}^{(n+1)}$, taking into account that $C^{(2 n+2)} P_{ \pm}^{(n+1)}=P_{ \pm}^{(n+1)} C^{(2 n+2)}$ and $\left(P_{ \pm}^{(n+1)}\right)^{2}=P_{ \pm}^{(n+1)}$, we obtain Eq. 411 where the projected equivalence matrix is equal to the product of all diagonal projected braid matrices and coincides with Eq. 42), which completes the proof of Proposition 2 .

Propositions 1 and 2 ultimately prove that the Pfaffian correlation functions with $2 n+2$ non-Abelian quasiholes at fixed positions indeed belong to one of the two inequivalent representations of the braid group $\mathscr{B}_{2 n+2}$ whose generators $B_{j}^{(2 n+2, \pm)}$ can be expressed as $\pi / 2$ rotations in terms of the $\operatorname{SO}(2 n+2) \gamma$-matrices and identifies the parity in the spinor representations with the fermion parity in the Ising model [7, 21, 6].

\section{Conclusions}

In this paper we have consistently derived the braid matrices representing the exchanges of 4 non-Abelian Ising anyons in both representations with positive and negative fermion parity. To this end we have used the fact that the braid matrices are independent of the distance between the braided particles, as well as the fusion rules for the Ising anyons in both Neveu-Schwarz and Ramond superselection sectors of the Ising model. In addition we found recurrence relations for the braid matrices $B_{j}^{(2 n+2, \pm)}$ for the exchanges of $2 n+2$ Ising anyons as well as explicit formulas for most of the braid generators in the representations with both parity. Finally, we have proven that the braid matrices derived from the multi-anyon Pfaffian wave functions are completely equivalent to the braid generators derived in the $\operatorname{SO}(2 n+2)$ spinor approach [7, 21] and have given explicitly the matrices establishing the equivalence in both representations.

\section{Acknowledgments}

I thank Ivan Todorov, Lyudmil Hadjiivanov and André Ahlbrecht for many useful discussions and Galileo Galilei Institute for Theoretical Physics in Firenze, Italy for hospitality as well as INFN for partial support. The author has been supported as a Research Fellow by the Alexander von Humboldt Foundation and by the BG-NCSR under Contract Nos. F-1406 and DO 02-257.

[1] S. D. Sarma, M. Freedman, and C. Nayak, "Topologically-protected qubits from a possible non-abelian fractional quantum Hall state," Phys. Rev. Lett. 94 (2005) 166802, cond-mat/0412343

[2] M. Freedman, C. Nayak, and K. Walker, "Towards universal topological quantum computation in the $v=5 / 2$ fractional quantum Hall state," Phys. Rev. B 73 (2006) 245307, arXiv: cond-mat/0512066

[3] C. Nayak, S. H. Simon, A. Stern, M. Freedman, and S. D. Sarma, "Non-Abelian anyons and topological quantum computation," Rev. Mod. Phys. 80 (2008) 1083-1159, arXiv:0707.1889

[4] G. Moore and N. Read, "Nonabelions in the fractional quantum Hall effect," Nucl. Phys. B360 (1991) 362-396.

[5] L. S. Georgiev, "Towards a universal set of topologically protected gates for quantum computation with Pfaffian qubits," Nucl. Phys. B 789 (2008) 552-590, hep-th/0611340 
[6] A. Ahlbrecht, L. S. Georgiev, and R. F. Werner, "Implementation of Clifford gates in the Ising-anyon topological quantum computer," Phys. Rev. A 79 (2009) 032311, arXiv:0812.2338

[7] C. Nayak and F. Wilczek, " $2 n$ quasihole states realize $2^{n-1}$-dimensional spinor braiding statistics in paired quantum Hall states," Nucl. Phys. B 479 (1996) 529-553, cond-mat/9605145

[8] J. K. Slingerland and F. A. Bais, "Quantum groups and nonabelian braiding in quantum Hall systems," Nucl. Phys. B 612 (2001) 229-290, arXiv: cond-mat/0104035v1

[9] D. Ivanov, "Non-Abelian statistics of half-quantum vortices in p-wave superconductors," Phys. Rev. Lett. 86 (2001) 268-271.

[10] N. Read and D. Green, "Paired states of fermions in two dimensions with breaking of parity and time-reversal symmetries and the fractional quantum Hall effect," Phys. Rev. B61 (2000) 10267-10297, cond-mat/9906453

[11] A. Stern, F. von Oppen, and E. Mariani, "Geometric phases and quantum entanglement as building blocks for non-Abelian quasiparticle statistics," Phys. Rev. B 70 (2004) 205338.

[12] V. Gurarie and C. Nayak, "A plasma analogy and Berry matrices for non-Abelian quantum Hall states," Nucl. Phys. B506 (1997) 685-694, arXiv: cond-mat/9706227

[13] S. H. Simon, "Effect of Landau level mixing on braiding statistics," Phys. Rev. Lett. 100 (2008) 116803, arXiv:0710.1088

[14] N. Read, "Non-abelian adiabatic statistics and Hall viscosity in quantum Hall states and $p_{x}+i p_{y}$ paired superfluids," Phys. Rev. B 79 (2009) 045308, arXiv: 0805.2507

[15] P. Furlan, G. Sotkov, and I. Todorov, "Two-dimensional conformal quantum field theory," Riv. Nuovo Cim. Vol. 12, No. 6 (1989) 1.

[16] L. S. Georgiev, "The $v=5 / 2$ quantum Hall state revisited: spontaneous breaking of the chiral fermion parity and phase transition between Abelian and non-Abelian statistics," Nucl. Phys. B 651 (2003) 331-360, hep-th/0108173

[17] P. Di Francesco, P. Mathieu, and D. Sénéchal, Conformal Field Theory. Springer-Verlag, New York, 1997.

[18] F. Wilczek and A. Zee, "Families from spinors," Phys. Rev. D 25 (Jan, 1982) 553-565.

[19] L. S. Georgiev, "Topologically protected gates for quantum computation with non-Abelian anyons in the Pfaffian quantum Hall state," Phys. Rev. B 74 (2006) 235112, cond-mat/0607125

[20] I. T. Todorov and Y. S. Stanev, Chiral Current Algebras and Two-dimensional Conformal Models. Troisieme Cycle de la Physique, En Swisse Romande, Université de Lausanne, 1992.

[21] L. S. Georgiev, "Computational equivalence of the two inequivalent spinor representations of the braid group in the topological quantum computer based on Ising anyons," (2008) arXiv:0812.2337

[22] L. S. Georgiev, "Topological quantum computation with the universal $R$ matrix for Ising anyons," in Proc. of the VII Int. Workshop "Lie Theory and its Applications in Physics", June 2007, Varna, Bulgaria, H.-D. Doebner and V. Dobrev, eds., pp. 256-265. Heron Press, Sofia, 2008. arXiv:0812.2333. 